\title{
Modeling Online Discourse with Coupled Distributed Topics
}

\author{
Nikita Srivatsan $^{\dagger} \quad$ Zachary Wojtowicz $^{\ddagger} \quad$ Taylor Berg-Kirkpatrick $^{\dagger}$ \\ ${ }^{\dagger}$ Language Technologies Institute, Carnegie Mellon University, \{asrivats, tberg\} @ cs. cmu .edu \\ ${ }^{\ddagger}$ Social and Decision Sciences, Carnegie Mellon University, zdw@ andrew. cmu . edu
}

\begin{abstract}
In this paper, we propose a deep, globally normalized topic model that incorporates structural relationships connecting documents in socially generated corpora, such as online forums. Our model (1) captures discursive interactions along observed reply links in addition to traditional topic information, and (2) incorporates latent distributed representations arranged in a deep architecture, which enables a GPU-based mean-field inference procedure that scales efficiently to large data. We apply our model to a new social media dataset consisting of $13 \mathrm{M}$ comments mined from the popular internet forum Reddit, a domain that poses significant challenges to models that do not account for relationships connecting user comments. We evaluate against existing methods across multiple metrics including perplexity and metadata prediction, and qualitatively analyze the learned interaction patterns.
\end{abstract}

\section{Introduction}

Topic models have become one of the most common unsupervised methods for uncovering latent semantic information in natural language data, and have found a wide variety of applications across the sciences. However, many common models such as Latent Dirichlet Allocation (Ng and Jordan, 2003) - make an explicit exchangeability assumption that treats documents as independent samples from a generative prior, thereby ignoring important aspects of text corpora which are generated by nonergodic, interconnected social systems. While the direct application of such models to datasets such as transcripts of The French Revolution (Barron et al., 2017) and discussions on Twitter (Zhao et al., 2011) have yielded sensible topics and exciting insights, their exclusion of document-to-document interactions imposes limitations on the scope of their applicability and the analyses they support.
For instance, on many social media platforms, comments are short (the average Reddit comment is 10 words long), making them difficult to treat as full documents, yet they do cohere as a collection, suggesting that contextual relationships should be considered. Moreover, analysis of social data is often principally concerned with understanding relationships between documents (such as question-asking and -answering), so a model able to capture such features is of direct scientific relevance.

To address these issues, we propose a design that models representations of comments jointly along observed reply links. Specifically, we attach a vector of latent binary variables to each comment in a collection of social data, which in turn connect to each other according to the observed reply-link structure of the dataset. The inferred representations can provide information about the rhetorical moves and linguistic elements that characterize an evolving discourse. An added benefit is that while previous work such as Sequential LDA (Du et al., 2012) has focused on modeling a linear progression, the model we present applies to a more general class of acyclic graphs such as tree-structured comment threads ubiquitous on the web.

Online data can be massive, which presents a scalability issue for traditional methods. Our approach uses latent binary variables similar to a Restricted Boltzmann Machine (RBM); related models such as Replicated Softmax (RS) (Salakhutdinov and Hinton, 2009) have previously seen success in capturing latent properties of language, and found substantial speedups over previous methods due to their GPU amenable training procedure. RS was also shown to deal well with documents of significantly different length, another key characteristic of online data. While RBMs permit exact inference, the additional coupling potentials present in our model make inference intractable. However, the choice of bilinear potentials and latent 


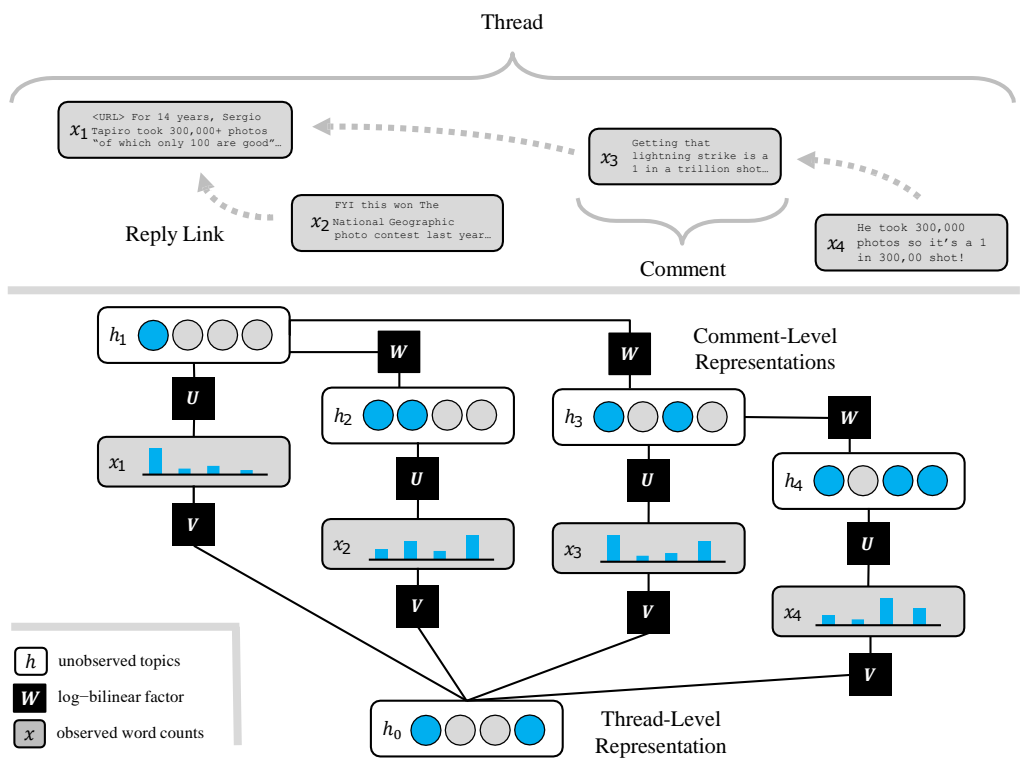

Figure 1: DDTM factor graph for an example thread. Each comment is modeled as an observed bag-of-words $x$ with topics represented by a latent binary vector $h$. Log-bilinear factors connect the latent and observed variables of each comment, and the latent variables of parent-child comment pairs along observed reply links. Biases are omitted for clarity.

features admits a mean-field inference procedure which takes the form of a series of dense matrix multiplications followed by nonlinearities, which is particularly amenable to GPU computation and lets us scale efficiently to large data.

Our model outperforms LDA and RS baselines on perplexity and downstream tasks including metadata prediction and document retrieval when evaluated on a new dataset mined from Reddit. We also qualitatively analyze the learned topics and discuss the social phenomena uncovered.

\section{Model}

We now present an overview of our model. Specifically, it will take the probabilistic form of an undirected graphical model whose architecture mirrors the tree structure of the threads in our data.

\subsection{Motivating Dataset}

We evaluate on a corpus mined from Reddit, an internet forum which ranks as the fourth most trafficked site in the US (Alexa, 2018) and sees millions of daily comments (Reddit, 2015). Discourse on Reddit follows a branching pattern, shown in Figure 1. The largest unit of discourse is a thread, beginning with a link to external content or a natural language prompt, posted to a relevant subreddit based on its subject matter. Users comment in response to the original post (OP), or to any other comment. The result is a structure which splits at many points into more specific or tangential discussions that while locally coherent may differ substantially from each other. The data reflect features of the underlying memory and network structure of the generating process; comments are serially correlated and highly cross-referential. We treat individual comments as "documents" under the standard topic modeling paradigm, but use observed reply structure to induce a tree of documents for every thread.

\subsection{Description of Discursive Distributed Topic Model}

We now introduce the Discursive Distributed Topic Model (DDTM) (illustrated in Figure 1). For each comment in the thread, DDTM assigns a latent vector of binary random variables (or bits) that collectively form a distributed embedding of the topical content of that comment; for instance, one bit might represent sarcastic language while another might track usage of specific acronyms - a given comment could have any combination of those features. These representations are tied to those of parent and child comments via coupling potentials (see Section 2.3), which allow them to learn discursive properties by inducing a deep undirected network over the thread. In order to encourage the model to use these comment-level representations to learn discursive and stylistic patterns as opposed to simply topics of discussion, we incorporate a single additional latent vector for the entire thread that interacts with each comment, explaining word choices that are mainly topical rather than discursive or stylistic. As we demonstrate in our experiments (see Section 6) the thread-level embedding learns distributions more reminiscent of what a traditional topic model would uncover, while the comment-level embeddings model styles of speak- 
ing and mannerisms that do not directly indicate specific subjects of conversation. The joint probability is defined in terms of an energy function that scores latent embeddings and observed word counts across the tree of comments within a thread using log-bilinear potentials, and is globally normalized over all word count and embedding combinations.

\subsection{Probability Model}

More formally, consider a thread containing $N$ comments each of size $D_{n}$ with a vocabulary of size $K$. As depicted in Figure 1, each comment is viewed as a bag-of-words, densely connected via a log-bilinear potential to a latent embedding of size $F$. Let each comment be represented as as an integer vector $x_{n} \in \mathbb{Z}^{K}$ where $x_{n k}$ is number of times word $k$ was observed in comment $n$, and let $h_{n}=\{0,1\}^{F}$ be the topic embedding for each comment, and let $h_{0}=\{0,1\}^{F}$ be the embedding for the entire thread. To model topic transitions, we score the embeddings of parent-child pairs with a separate coupling potential as shown in Figure 1 (comments with no parents or children receive additional start/stop biases respectively). Let replies be represented with sets $R, P_{N}$, and $C_{N}$ where $(n, m) \in R$ and $n \in P_{m}$ and $m \in C_{n}$ if comment $m$ is a reply to comment $n$. DDTM assigns probability to a specific configuration of $x, h$ with an energy function scored by the emission $\left(\pi_{\mathrm{e}}\right)$ and coupling $\left(\pi_{\mathrm{c}}\right)$ potentials.

$$
\begin{aligned}
E(x, h ; \theta) & =\underbrace{\sum_{n=1}^{N} \pi_{\mathrm{e}}(h, x, n)}_{\text {Emission Potentials }}+\underbrace{\sum_{(n, m) \in R} \pi_{\mathrm{c}}(h, n, m)}_{\text {Coupling Potentials }} \\
\pi_{\mathrm{e}}(h, x, n) & =h_{n}^{\top} U x_{n}+x_{n}^{\top} a+D_{n} h_{n}^{\top} b \\
& +h_{0}^{\top} V x_{n}+D_{n} h_{0}^{\top} c \\
\pi_{\mathrm{c}}(h, n, m) & =h_{n}^{\top} W h_{m}
\end{aligned}
$$

Note that the bias on embeddings is scaled by the number of words in the comment, which controls for their highly variable length. The joint probability is computed by exponentiating the energy and dividing by a normalizing constant.

$$
\begin{aligned}
p(x, h ; \theta) & =\frac{\exp (E(x, h ; \theta))}{Z(\theta)} \\
Z(\theta) & =\sum_{x^{\prime}, h^{\prime}} \exp \left(E\left(x^{\prime}, h^{\prime} ; \theta\right)\right)
\end{aligned}
$$

This architecture encourages the model to learn discursive maneuvers via the coupling potentials while separating within-thread variance and acrossthread variance through the comment-level and thread-level embeddings respectively. The coupling of latent variables makes factored inference impossible, meaning that even the exact computation of the partition function is no longer tractable. This necessitates approximating the gradients for learning which we will now address.

\section{Learning and Inference}

Inference in this model class in intractable, so as has been done in previous work on topic modeling (Ng and Jordan, 2003) we rely on variational methods to approximate the gradients needed during training as well as the posteriors over the topic bit vectors. Specifically, we will need the gradients of the normalizer and the sum of the energy function over the hidden variables

$$
E(x ; \theta)=\log \sum_{h} \exp (E(x, h ; \theta))
$$

which we refer to as the marginal energy. Following the approach described for undirected models by Eisner (2011), we approximate these quantities and their gradients with respect to the model parameters $\theta$ as we will now describe (thread-level embeddings are omitted in this section for clarity).

\subsection{Normalizer Approximation}

We aim to train our model to maximize the marginal likelihood of the observed comment word counts, conditioned on the reply links. To do this we must compute the gradient of the normalizer $Z(\theta)$. However, this quantity is computationally intractable, as it contains a summation over all exponential choices for every word in the thread. Therefore, we must approximate $Z(\theta)$. Observe that under Jensen's Inequality, we can form the following lower bound on the normalizer using an approximate joint distribution $q^{(\mathrm{Z})}$.

$$
\begin{aligned}
& \log Z(\theta)=\log \sum_{x, h} \exp (E(x, h ; \theta)) \\
& \geq \mathbb{E}_{q^{(\mathrm{Z})}}[E(x, h ; \theta)]-\mathbb{E}_{q^{(\mathrm{Z})}}\left[\log q^{(\mathrm{Z})}(x, h ; \phi, \gamma)\right]
\end{aligned}
$$

We now define $q^{(\mathrm{Z})}$ as depicted in Figure 2 as a mean-field approximation that treats all variables as independent. We parameterize $q^{(\mathrm{Z})}$ with 


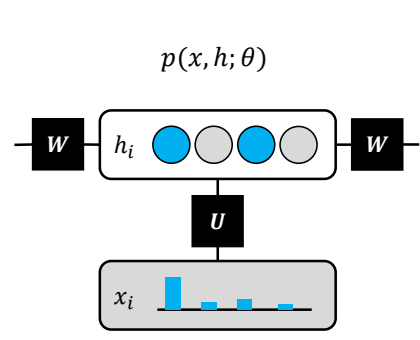

Joint Probability
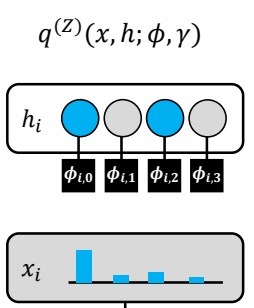

$\gamma_{i}$

Variational Approx. to Joint

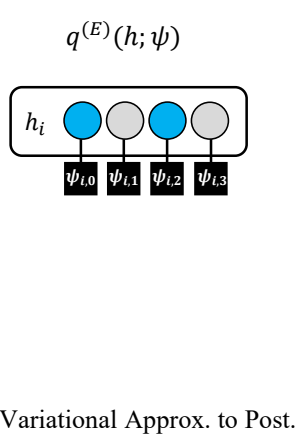

Figure 2: Factor graph of full joint compared to meanfield approximations to joint and posterior. $\phi_{n f} \in[0,1]$, independent Bernoulli parameters representing the probability of $h_{n f}$ being equal to 1 , and $\gamma_{n k}$ replicated softmaxes representing the probability of a word in $x_{n}$ taking the value $k$. Note that all words in $x_{n}$ are modeled as samples from this single distribution. The approximation then factors as follows:

$$
\begin{aligned}
& q^{(\mathrm{Z})}(x, h ; \phi, \gamma)=q^{(\mathrm{Z})}(x ; \gamma) \cdot q^{(\mathrm{Z})}(h ; \phi) \\
& q^{(\mathrm{Z})}(x ; \gamma)=\prod_{n=1}^{N} \prod_{k=1}^{K}\left(\gamma_{n k}\right)^{x_{n k}} \\
& q^{(\mathrm{Z})}(h ; \phi)=\prod_{n=1}^{N} \prod_{f=1}^{F}\left(\left(\phi_{n f}\right)^{h_{n f}}\left(1-\phi_{n f}\right)^{\left(1-h_{n f}\right)}\right)
\end{aligned}
$$

We optimize the parameters of $q^{(\mathrm{Z})}$ to maximize its variational lower bound, via iterative mean-field updates, which allow us to perform coordinate ascent over the parameters of $q^{(\mathrm{Z})}$. Maximizing the lower bound with respect to particular $\phi_{n f}$ and $\gamma_{n k}$ while holding all other parameters frozen, yields the following mean-field update equations (biases omitted for clarity):

$$
\begin{aligned}
& \phi_{n .}=\sigma\left(U \gamma_{n}+\sum_{m \in C_{n}} W \phi_{m}+\phi_{P_{n}}^{\top} W\right) \\
& \gamma_{n .}=\sigma\left(\phi_{n}^{\top} U\right)
\end{aligned}
$$

We iterate over the parameters of $q^{(\mathrm{Z})}$ in an "upward-downward" manner; first updating $\phi$ for all comments with no children, then all comments whose children have been updated, and so on up to the root of the thread. Then we perform the same updates in reverse order. After updating all $\phi$, we then update $\gamma$ simultaneously (the components of $\gamma$ are independent conditioned on $\phi$ ). We iterate these upward-downward passes until convergence.

\subsection{Marginal Energy Approximation}

We can now approximate the normalizer, but still need the marginal data likelihood in order to take gradient steps on it and train our model. In order to recover the marginal likelihood, we must next approximate the marginal energy $E(x ; \theta)$ as it too is intractable. This is due to the coupling potentials, which make the topics across comments dependent even when conditioned on the word counts. To do this, we form an additional variational approximation (see Figure 2) to the marginal energy, which we optimize similarly.

$$
\begin{aligned}
& E(x ; \theta)=\log \sum_{h} \exp (E(x, h ; \theta)) \\
& \geq \mathbb{E}_{q^{(\mathrm{E})}}[E(x, h ; \theta)]-\mathbb{E}_{q^{(\mathrm{E})}}\left[\log q^{(\mathrm{E})}(h ; \psi)\right]
\end{aligned}
$$

Since $q^{(\mathrm{E})}(h ; \psi)$ need only model the hidden units $h$, we can parameterize it in the same manner as $q^{(\mathrm{Z})}(h ; \phi)$. Note that while these distributions factor similarly, they do not share parameters, although we find that in practice, initializing $\phi \leftarrow \psi$ improves our approximation. We optimize the lower bound on $E(x ; \theta)$ via a similar coordinate ascent strategy, where the mean-field updates take the following form (biases omitted for clarity):

$$
\psi_{n .}=\sigma\left(U h_{n}+\sum_{m \in C_{n}} W \psi_{m}+\psi_{P_{n}}^{\top} W\right)
$$

We can use $q^{(\mathrm{E})}$ to perform inference at test time in our model, as its parameters $\psi$ directly correspond to the expected values of the hidden topic embeddings under our approximation.

\subsection{Learning via Gradient Ascent}

We train the parameters of our true model $p(x, h ; \theta)$ via stochastic updates wherein we optimize both ap- 
proximations on a single datum (i.e. thread) to compute the approximate gradient of its log-likelihood, and take a single gradient step on the model parameters (repeating on all training instances until convergence). That gradient is given by the difference in feature expectations under the approximations (entropy terms from the lower bounds are dropped as they do not depend on $\theta$ ).

$$
\begin{aligned}
\nabla \log p(x ; \theta) & \approx \mathbb{E}_{q^{(\mathrm{E})}(h ; \psi)}[\nabla E(x, h ; \theta)] \\
& -\mathbb{E}_{q^{(\mathrm{Z})}\left(x^{\prime}, h ; \psi\right)}\left[\nabla E\left(x^{\prime}, h ; \theta\right)\right]
\end{aligned}
$$

In summary, we use two separate mean-field approximations to compute lower bounds on the marginal energy $E(x, h ; \theta)$, and its normalizer $Z(\theta)$, which lets us approximate the marginal likelihood $p(x ; \theta)$. Note that as our estimate on the marginal likelihood is the difference between two lower bounds, it is not a lower bound itself, although in practice it works well for training.

\subsection{Scalability and GPU Implementation}

Given the magnitude of our dataset, it is essential to be able to train efficiently at scale. Many commonly used topic models such as LDA ( $\mathrm{Ng}$ and Jordan, 2003) have difficulty scaling, particularly if trained via MCMC methods. Improvements have been shown from online training (Hoffman et al., 2010), but extending such techniques to model comment-to-comment connections and leverage GPU compute is nontrivial.

In contrast, our proposed model and mean-field procedure can be scaled efficiently to large data because they are amenable to GPU implementation. Specifically, the described inference procedure can be viewed as the output of a neural network. This is because DDTM is globally normalized with edges parameterized as log-bilinear weights, which results in the mean-field updates taking the form of matrix operations followed by nonlinearities. Therefore, a single iteration of mean-field is equivalent to a forward pass through a recursive neural network, whose architecture is defined by the tree structure of the thread. Multiple iterations are equivalent to feeding the output of the network back into itself in a recurrent manner, and optimizing for $T$ iterations is achieved by unrolling the network over $T$ timesteps. This property makes DDTM highly amenable to efficient training on a GPU, and allowed us to scale experiments to a dataset of over $13 \mathrm{M}$ total Reddit comments.

\section{Experimental Setup}

\subsection{Data}

We mined a corpus of Reddit threads pulled through the platform's API. Focusing on the twenty most popular subreddits (gifs, todayilearned, CFB, funny, aww, AskReddit, BlackPeopleTwitter, videos, pics, politics, The_Donald, soccer, leagueoflegends, nba, nfl, worldnews, movies, mildlyinteresting, news, gaming) over a one month period yielded 200, 000 threads consisting of $13,276,455$ comments total. The data was preprocessed by removing special characters, replacing URLs with a domain-specific token, stemming English words using a Snowball English Stemmer (Porter, 2001), removing stopwords, and truncating the vocabulary to only include the top 10,000 most common words. OPs are modeled as a comment at the root of each thread to which all top-level comments respond. This dataset will be made available for public use after publication.

\subsection{Baselines and Comparisons}

We compare to baselines of Replicated Softmax (RS) (Salakhutdinov and Hinton, 2009) and Latent Dirichlet Allocation (LDA) (Ng and Jordan, 2003). RS is a distributed topic model similar to our own, albeit without any coupling potentials. LDA is a locally normalized topic model which defines topics as non-overlapping distributions over words. To ensure that DDTM does not gain an unfair advantage purely by having a larger embedding space, we divide the dimensions equally between commentand thread-level. Unless specified 64 bits/topics were used. We experiment with RS and LDA treating either comments or full threads as documents.

\subsection{Training and Initialization}

SGD was performed using the Adam optimizer (Kingma and $\mathrm{Ba}, 2015$ ). When running inference, we found convergence was reached in an average of 2 iterations of updates. Using a single NVIDIA Titan X (Pascal) card, we were able to train our model to convergence on the training set of $10 \mathrm{M}$ comments in less than 30 hours. It is worth noting that we found DDTM to be fairly sensitive to initialization. We found best results from Gaussian noise, with comment-level emissions at variance of 0.01 , thread-level emissions at 0.0001 , and transitions at 0 . We initialized all biases to 0 except for the bias on word counts, which we set to the unigram log-probabilities from the train set. 


\begin{tabular}{lcccc}
\hline & \multicolumn{4}{c}{ Perplexity (nats) } \\
Bits & 32 & 64 & 96 & 128 \\
\hline RS (thr) & 2240 & 2234 & 2233 & 2257 \\
RS (cmt) & 1675 & 1894 & 2245 & 2518 \\
\hline DDTM (-cpl) & 2027 & 1704 & 1766 & 1953 \\
DDTM & $\mathbf{1 6 2 4}$ & $\mathbf{1 5 9 0}$ & $\mathbf{1 7 1 9}$ & $\mathbf{7 1 3}$ \\
\hline
\end{tabular}

Table 1: Perplexity of DDTM with and without coupling potentials (-cpl) vs. baselines trained at comment (cmt) or thread (thr) level across various numbers of topics and bits. For reference, a unigram model achieves 2644 .

\section{Results}

\subsection{Evaluating Perplexity}

We compare models by perplexity on a held-out test set, a standard evaluation for generative and latent variables models.

Setup: Due to the use of mean-field approximations for both the marginal energy and normalizer we lose any guarantees regarding the accuracy of our likelihood estimate (both approximations are lower bounds, and therefore their difference is neither a strict lower bound nor guaranteed to be unbiased). To evaluate perplexity in a more principled way, we use Annealed Importance Sampling (AIS) to estimate the ratio between our model's normalizer and the tractable normalizer of a base model from which we can draw true independent samples as described by Salakhutdinov and Murray (2008). Note that since the marginal energy is intractable in our model, unlike a standard RBM, we must sample the joint - and not the marginal - intermediate distributions. This yields an unbiased estimate of the normalizer. The marginal energy must still be approximated via a lower bound, but given that AIS is unbiased and empirically low in variance, we can treat the overall estimate as a lower bound on likelihood for evaluation. Using 2000 intermediate distributions, and averaging over 20 runs, we evaluated per-word perplexity over a set of 50 unseen threads. Results are shown in Table 1.

Results: DDTM achieves the lowest perplexity at all dimensionalities. Note our ablation with the coupling potentials removed (-cpl), increases perplexity noticeably, indicating that modeling replies helps beyond simply modeling threads and comments jointly, particularly at larger embeddings. For reference, a unigram model achieves 2644.

\begin{tabular}{lcc}
\hline Task & $\begin{array}{c}\text { Upvote Regr. } \\
\text { (MSE) }\end{array}$ & $\begin{array}{c}\text { Deletion Pred. } \\
(\% \text { acc. })\end{array}$ \\
\hline LDA (thr) & 1.952 & 68.35 \\
LDA (cmt) & 2.047 & 59.26 \\
\hline RS (thr) & 2.024 & 69.92 \\
RS (cmt) & 2.007 & 66.45 \\
\hline DDTM & $\mathbf{1 . 9 3 3}$ & $\mathbf{7 0 . 3 9}$ \\
\hline
\end{tabular}

Table 2: Performance of DDTM vs. Replicated Softmax (RS) and Latent Dirichlet Allocation (LDA) at predicting upvotes and child deletion.

We find that LDA's approximate perplexity is even worse, likely due to slackness in its lower bound.

\subsection{Upvote Regression}

To measure how well embeddings capture comment-level characteristics, we feed them into a linear regression model that predicts the number of upvotes the comment received. Upvotes provide a loose human-annotated measure of likability. We expect that context matters in determining how well received a comment is; the same comment posted in response to different parents may receive a very different number of upvotes. Hence, we expect comment-level embeddings to be more informative for this task when connected via our model's coupling potentials.

Setup: We trained a standard linear regressor for each model. The regressor was trained using ordinary least squares on the entire training set of comments using the model's computed topic embeddings as input, and the number of upvotes on the comment as the output to predict. As a preprocessing step, we took the log of the absolute number of votes before training. We compared models by mean squared error (MSE) on our test set. Results are shown in Table 2.

Results: DDTM achieves lowest MSE. To assess statistical significance, we performed a 500 sample bootstrap of our training set. The standard errors of these replications are small, and a two-sample t-test rejects the null hypothesis that DDTM has an average MSE equal to that of the next best method $(p<.001)$. Note that our model outperforms both comment- and thread-level embeddings, suggesting that modeling these jointly, and modeling the effect of neighboring representations in the comment graph, more accurately learns information relevant to a comment's social impact. 


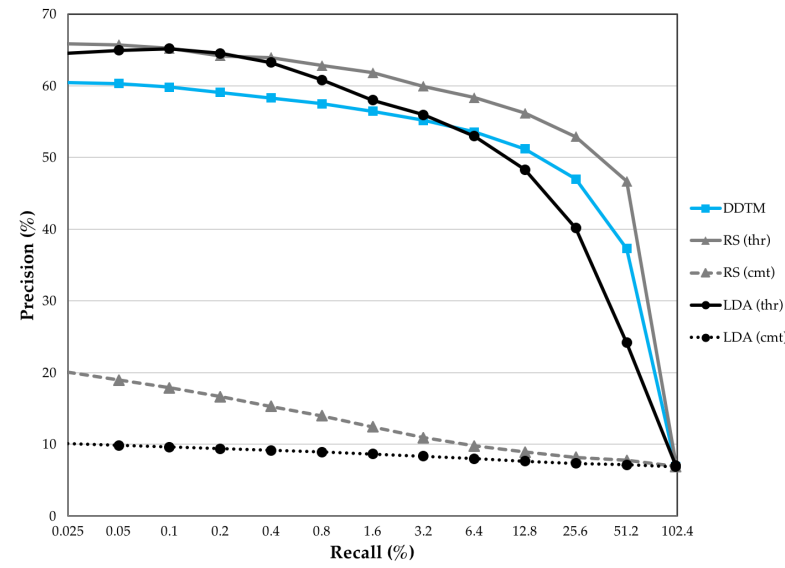

Figure 3: Precision vs. recall for document retrieval based on subreddit comparing various models for 1000 randomly selected held-out query comments.

\subsection{Deletion Prediction}

Comments that are excessively provocative or in violation of site rules are often deleted, either by the author or a moderator. We can measure whether DDTM captures discursive interactions that lead to such intervention by training a logistic classifier that predicts whether any of a given comment's children have been deleted.

Setup: For each model, a logistic regression classifier was trained stochastically with the Adam optimizer on the entire training set of comments using the model's computed topic embeddings as input, and a binary label for whether the comment had any deleted children as the output to predict. We compared models by accuracy on our test set. Results are shown in Table 2.

Results: DDTM gets the highest accuracy. Interestingly, thread-level models do better than comment-level ones, which suggests that certain topics or even subreddits may correlate with comments being deleted. This makes sense given that subreddits vary in severity of moderation. DDTM's performance also demonstrates that modeling comment-to-comment interaction patterns is helpful in predicting when a comment will spawn a deleted future response, which strongly matches our intuition.

\subsection{Document Retrieval}

Finally, while DDTM is not designed to better capture topical structure, we evaluate the extent to which it can still capture this information by performing document retrieval, a standard evaluation, for which we treat the subreddit to which a thread

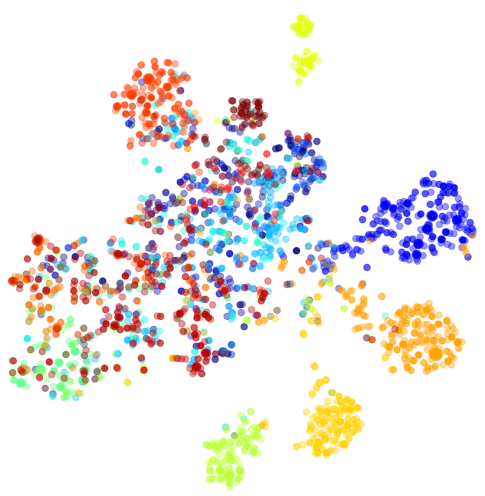

Figure 4: t-SNE visualization of a random sample of DDTM thread-level embeddings colored by subreddit (not observed in training)

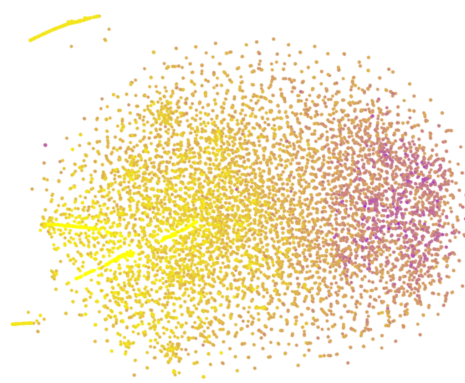

Figure 5: t-SNE visualization of a random sample of DDTM comment-level embeddings colored by log of comment length (darker is longer).

was posted as a label for relevance. Note that every comment within the same thread belongs to the same subreddit, which gives thread-level models an inherent advantage at this task. We include this task purely for the purpose of demonstrating that by capturing discursive patterns, DDTM does not lose the ability to model thread-level topics as well. Setup: Given a query comment from our held-out test set, we rank the training set by the Dice similarity of the hidden embeddings computed by the model. We consider a retrieved comment relevant to the query if they both originate from the same subreddit, which loosely categorizes the semantic content. Tuning the number of documents we return allows us to form precision recall curves, which we show in Figure 3.

Results: DDTM outperforms both comment-level baselines and is competitive with thread-level models, even beating LDA at high levels of recall. This indicates that despite using half of its dimensions to model comment-to-comment interactions DDTM can still do almost as good a job of modeling threadlevel semantics as a model using its entire capacity 


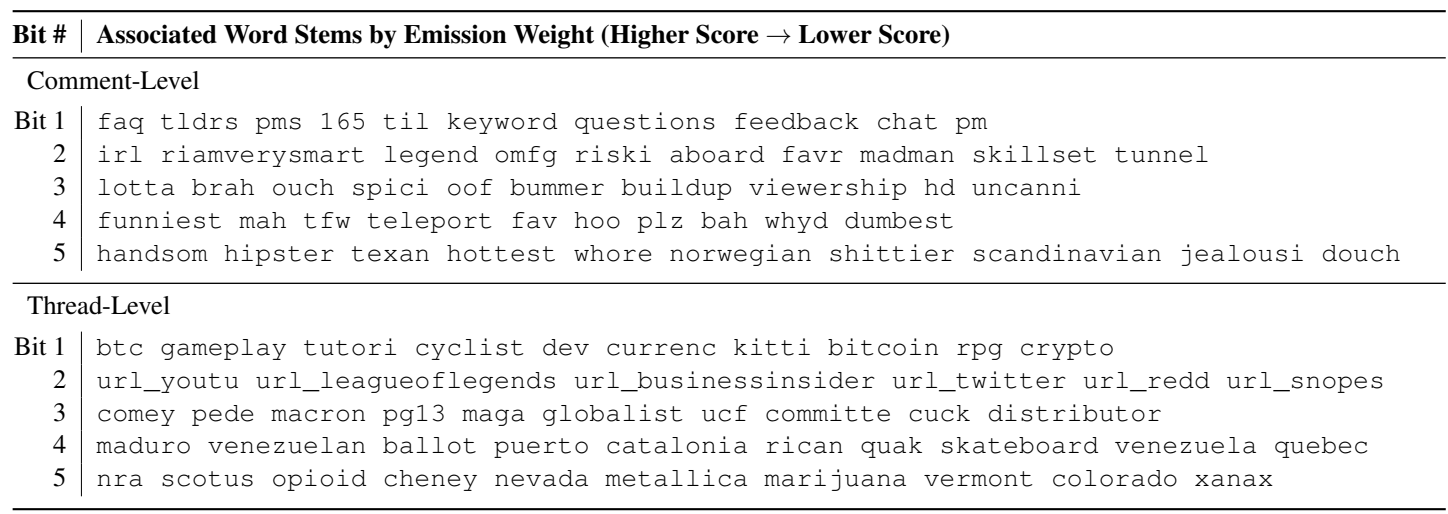

Table 3: Words with the highest emission weight for various comment-level and thread-level bits.

to do so. The gap between comment-level RS and LDA is also consistent with LDA's known issues dealing with sparse data (Sridhar, 2015), and lends credence to our theory that distributed topic representations are better suited to such domains.

\section{Qualitative Analysis of Topics}

We now offer qualitative analysis of the topic embeddings learned by our model. Note that since we use distributed embeddings, our bits are more akin to filters than complete distributions over words, and we typically observe as many as half of them active for a single comment. In a sense, we have an exponential number of topics, whose parameterization simply factors over the bits. Therefore, it can be difficult to interpret them as one would interpret topics learned by a model such as LDA. Furthermore, we find that in practice this effect is correlated with the topic embedding size; the more bits our model has, the less sparse and consequently less individually meaningful the bits become. Therefore for this analysis, we specifically focus on DDTM trained with 64 bits total.

\subsection{Bits in Isolation}

Directly inspecting the emission parameters, reveals that the comment-level and thread-level halves of our embeddings capture substantially different aspects of the data (shown in Table 3) akin to vertical, within-thread, and horizontal, across-thread sources of variance respectively. The comment-level topic bits tend to reflect styles of speaking, lingo, and memes that are not unique to a particular subject of discourse or even subreddit. For example, comment-level Bit 2 captures many words typical of taunting Reddit comments; replying with "/r/iamverysmart" (a subreddit dedicated to mocking people who make grandiose claims about their intellect) is a common way of jokingly implying that the author of the parent comment takes themselves too seriously - and thus corresponds to a certain kind of rhetorical move. Further, it is grouped with other words that indicate related rhetorical moves; calling a user "risky" or a "madman" is a common means of suggesting that they are engaging in a pointless act of rebellion. They also cluster at the coarsest level by length (see Figure 5) which we find to correlate with writing style.

By contrast, the thread-level bits are more indicative of specific topics of discussion, and unsurprisingly they cluster by subreddit (see Figure 4). For example, thread-level Bit 3 captures lexicon used almost exclusively by alt-right Donald Trump supporters as well as the names of various political figures. Bit 4 highlights words related to civil unrest in Spanish speaking parts of the world.

\subsection{Bits in Combination}

While these distributions over words (particularly for comment-level bits) can seem vague, when multiple bits are active, their effects compound to produce much more specific topics. One can think of the bits as defining soft filters over the space of words, that when stacked together carve out patterns not apparent in any of them individually. We now analyze a few sample topic embeddings. To do this, we perform inference as described on a held-out thread, and pass the comment-level topic embedding for a single sampled comment through our emission matrix and inspect the words with the highest corresponding weight (shown in Table 4). In generative terminology, these can be thought of as reconstructions of comments.

These topic embeddings capture more specific conversational and rhetorical moves. For example, 


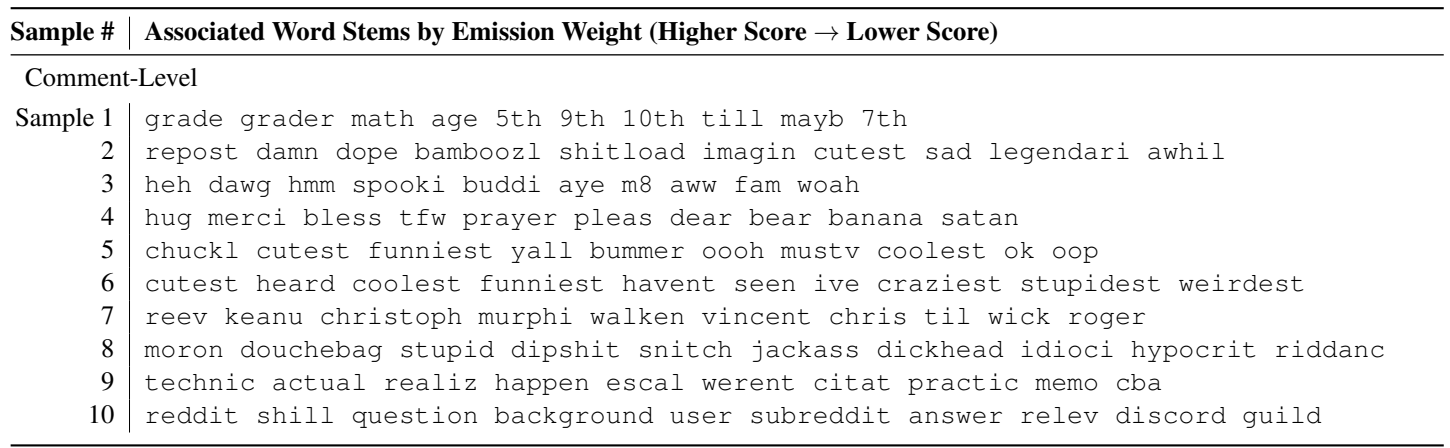

Table 4: Words with the highest emission weight for sample held-out comment reconstructions.

Sample 6 displays supportive and interested reactionary language, which one might expect to see used in response to a post or comment linking to media or describing something intriguing. This is of note given that one of the primary aims of including coupling potentials was to encourage DDTM to learn "topics" that correspond to responses and interactive behavior, something existing methods are largely not designed for. By contrast, Sample 9 captures a variety of hostile language and insults, which unlike those discussed previously do not denote membership in a particular online community. As patterns of toxic and hateful behavior on Reddit are more well-studied (Chandrasekharan et al., 2017), it could be useful to have a tool to analyze precipitous contexts and parent comments, something which we hope systems based on coupling of comment embeddings have the capacity to provide. Sample 10 is of particular interest as it consists largely of Reddit terminology. Conversations about the meta of the site can manifest for example in users accusing each other of being "shills" (i.e. accounts paid to astroturf on behalf of external interests) or requesting/responding to "guilding", a feature which lets users purchase premium access for each other often in response to a particularly well made comment.

\section{Related Work}

Many topic models such as LDA ( $\mathrm{Ng}$ and Jordan, 2003) treat documents as independent mixtures, yet this approach fails to model how comments interact with one another throughout a larger discourse if such connections exist in the data. Other work has considered modeling hierarchy in topics (Griffiths et al., 2004). These models form hierarchical representations of topics themselves, but still treat documents as independent. While this approach can succeed in learning topics of various granulari- ties, it does not explicitly track how topics interact in the context of a nested conversation.

Some approaches such as Pairwise-Link-LDA and Link-PSLA-LDA (Nallapati et al., 2008) attempt to model interactions among documents in an arbitrary graph, albeit with important drawbacks. The former models every possible pairwise link between comments, and the latter models links as a bipartite graph, limiting its ability to scale to large tree-structured threads. Similar work on Topic-Link LDA (Liu et al., 2009) models link probabilities conditioned on both topic similarity and an authorship model, yet this approach is poorly suited to high volume, semi-anonymous online domains. Other studies have leveraged replystructures on Reddit in the context of predicting persuasion (Hidey and McKeown, 2018), but DDTM differs in its generative, unsupervised approach.

DDTM's emission potentials are similar to those of Replicated Softmax (Salakhutdinov and Hinton, 2009), an undirected model based on a Restricted Boltzmann Machine. Unlike LDA-style models, RS does not assign a topic to each word, but instead builds a distributed representation. In this setting, a single word can be likely under two different topics, both of which are present, and lend probability mass to that word. LDA-style models by contrast would require the topics to compete for the word.

\section{Conclusion}

In this paper we introduce a novel way to learn topic interactions in observed discourse trees, and describe GPU-amenable learning techniques to train on large-scale data mined from Reddit. We demonstrate improvements over previous models on perplexity and downstream tasks, and offer qualitative analysis of learned discursive patterns. The dichotomy between the two levels of embeddings hints at applications in style-transfer. 


\section{References}

Alexa. 2018. Reddit.com traffic, demographics and competitors. https://www.alexa.com/ siteinfo/reddit.com. Accessed: 2018-0222.

Alexander TJ Barron, Jenny Huang, Rebecca L Spang, and Simon DeDeo. 2017. Individuals, institutions, and innovation in the debates of the french revolution. arXiv preprint arXiv: 1710.06867 .

Eshwar Chandrasekharan, Umashanthi Pavalanathan, Anirudh Srinivasan, Adam Glynn, Jacob Eisenstein, and Eric Gilbert. 2017. You can't stay here: The efficacy of reddit's 2015 ban examined through hate speech. Proceedings of the ACM on HumanComputer Interaction, $1(\mathrm{CSCW}): 31$.

Lan Du, Wray Buntine, Huidong Jin, and Changyou Chen. 2012. Sequential latent dirichlet allocation. Knowledge and information systems, 31(3):475503.

Jason Eisner. 2011. High-level explanation of variational inference. https://www.cs.jhu.edu/ jason/tutorials/variational. html. Accessed: 2018-02-22.

Thomas L Griffiths, Michael I Jordan, Joshua B Tenenbaum, and David M Blei. 2004. Hierarchical topic models and the nested chinese restaurant process. In Advances in neural information processing systems, pages 17-24.

Christopher Hidey and Kathleen McKeown. 2018. Persuasive influence detection: The role of argument sequencing. In Association for the Advancement of Artificial Intelligence.

M. Hoffman, D. Blei, and F. Bach. 2010. Online learning for latent dirichlet allocation. Neural Information Processing Systems.

Diederik P. Kingma and Jimmy Ba. 2015. Adam: A method for stochastic optimization. 3rd International Conference for Learning Representations.

Yan Liu, Alexandru Niculescu-Mizil, and Wojciech Gryc. 2009. Topic-link lda: joint models of topic and author community. In proceedings of the 26th annual international conference on machine learning, pages 665-672. ACM.

Ramesh M Nallapati, Amr Ahmed, Eric P Xing, and William W Cohen. 2008. Joint latent topic models for text and citations. In Proceedings of the 14th ACM SIGKDD international conference on Knowledge discovery and data mining, pages 542-550. ACM.

D. Blei A. Ng and M. Jordan. 2003. Latent dirichlet allocation. Journal of Machine Learning Research, 3:993-1022.
Martin Porter. 2001. Snowball: A language for stem-ming algorithms. http://snowball. tartarus. org/texts. Accessed: 2018-02-22.

Reddit. 2015. Reddit in 2015. https : //redditblog.com/2015/12/31/ reddit-in-2015/. Accessed: 2018-02-22.

Ruslan Salakhutdinov and Geoffrey Hinton. 2009. Replicated softmax: An undirected topic model. Advances in Neural Information Processing Systems, 22:1607-1614.

Ruslan Salakhutdinov and Iain Murray. 2008. On the quantitative analysis of deep belief networks. Proceedings of the 25th International Conference on Machine Learning.

Vivek Kumar Rangarajan Sridhar. 2015. Unsupervised topic modeling for short texts using distributed representations of words. In Proceedings of the 1st Workshop on Vector Space Modeling for Natural Language Processing, pages 192-200.

Wayne Xin Zhao, Jing Jiang, Jianshu Weng, Jing He, Ee-Peng Lim, Hongfei Yan, and Xiaoming Li. 2011. Comparing twitter and traditional media using topic models. In European Conference on Information Retrieval, pages 338-349. Springer. 